\title{
SUR LA COMPARAISON DES NOYAUX DE TATE D'ORDRE SUPÉRIEUR
}

\author{
par
}

David Vauclair

\begin{abstract}
Résumé. - We study generalized Tate kernels attached to a number field. It is known that there is a relationship between the equality of two of them and the size of some capitulation kernel in cyclotomic Iwasawa theory. By introducing some finer "Tate kernels $\bmod p^{k}$ ", we are able to describe this relationship very precisely, and to understand its asymptotic nature.
\end{abstract}

\section{Table des matières}

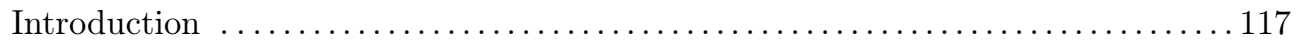

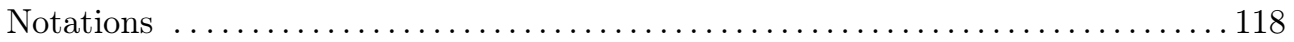

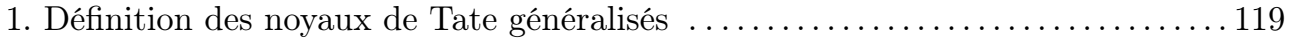

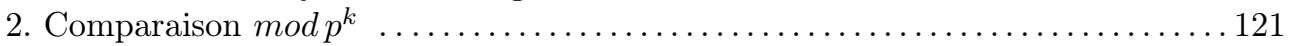

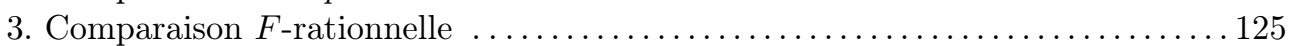

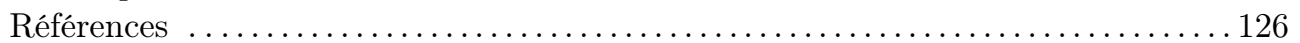

\section{Introduction}

Soit $p$ un nombre premier impair, et $F$ un corps de nombres. Supposons d'abord que $F$ contienne une racine primitive $p^{\text {ième }}$ de l'unité, notée $\zeta_{p}$. Dans $[\mathbf{G}]$ (voir aussi $[\mathbf{K C}]$ ), l'auteur fait l'étude des deux sous-groupes suivants de $F^{\times}$:

- $D^{(0)}(F)$, formé des éléments $x \in F^{\times}$dont l'extension de Kummer associée $\left.F^{p} \sqrt{x}\right) / F$ est soit triviale soit le premier étage d'une $\mathbb{Z}_{p}$-extension.

- le noyau de Tate $D^{(2)}(F)$ formé des éléments $x \in F^{\times}$pour lesquels le symbole de Steinberg $\left\{x, \zeta_{p}\right\} \in K_{2} F$ est trivial.

Sous la conjecture de Leopoldt, on sait que l'image de ces deux groupes dans $F^{\times} / F^{\times p}$ possède le même ordre. Aussi J. Coates avait-il posé la question suivante, rapportée dans $[\mathbf{G}]$ :

Question 0.1. - Supposons la conjecture de Leopoldt vraie pour $F$ et p. A-t-on alors $D^{(0)}(F)=$ $D^{(2)}(F)$ ?

R. Greenberg (loc. cit.) a montré que la réponse à cette question dépend de $F$. Si le noyau de capitulation asymptotique dans la $\mathbb{Z}_{p}$-extension cyclotomique de $F$ (noté $X^{\prime}\left(F_{\infty}\right)^{0}$ ) est trivial, alors l'égalité a lieu; cela se produit par exemple pour $F=\mathbb{Q}\left(\mu_{p}\right)$, sous la conjecture de Vandiver. Greenberg produit aussi une condition explicite (qu'il vérifie pour $F=\mathbb{Q}(\sqrt{-3.257})$ ) sous laquelle l'égalité n'a pas lieu.

Ces résultats mettent en évidence le fait suivant : la "différence" entre $D^{(2)}$ et $D^{(0)}$ est "contrôlée" par le noyau de capitulation. Ce travail vise à préciser le sens de la phrase précédente. Pour cela,

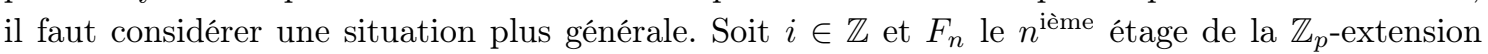
cyclotomique $F_{\infty} / F$; on ne suppose plus $\zeta_{p} \in F$. Nous introduisons des noyaux de Tate généralisés 
$\bmod p^{k}$, notés $V_{i}\left(F_{n}, p^{k}\right)$. Si $\zeta_{p} \in F$, ils sont tels que $V_{i}\left(F_{n}, p\right)=D^{(i)}\left(F_{n}\right) / F_{n}^{\times p}$ pour $i=0,2$ et plus généralement pour $i \neq 1$, si l'on prend pour $D^{(i)}\left(F_{n}\right)$ la définition adoptée par [KM], [AM] et $[\mathbf{H}]$. Le cas $i=1$ demande un traitement particulier ( $\mathrm{cf}[\mathbf{H}]$ et $[\mathbf{V 2}])$, et nous l'éviterons ici.

Fixons un corps de base $F$. Le problème de la comparaison de $V_{i}\left(F_{n}, p^{k}\right)$ avec $V_{j}\left(F_{n}, p^{k}\right)$ a un sens dès que $i \equiv j \bmod \left[F_{n}\left(\mu_{p^{k}}\right): F_{n}\right]$. Sous cette hypothèse, notre résultat principal (cf 2.2) met en relation les quantités suivantes :

- le nombre de racines de l'unité contenues dans $F_{n}$; on note $m\left(F_{n}\right)=\max \left\{m, \mu_{p^{m}} \subset F_{n}\left(\mu_{p}\right)\right\}$.

- la distance $p$-adique entre $i$ et $j$.

- on suppose que les groupes $X^{(i)}\left(F_{\infty}\right)^{\Gamma_{n}}=X^{\prime}\left(F_{\infty}\right)(i-1)^{\Gamma_{n}}$ et $X^{(j)}\left(F_{\infty}\right)^{\Gamma_{n}}=X^{\prime}\left(F_{\infty}\right)(j-1)^{\Gamma_{n}}$ (cf notations) sont finis, i.e. que $i$ et $j$ sont $F_{n}$ bons (cf 1.7), et l'on note $h\left(F_{n}, i, j\right)$ le minimum de leurs deux exposants.

- l'entier $k=k\left(F_{n}, i, j\right) \leq v_{p}(i-j)+m\left(F_{n}\right)$ maximal pour lequel l'égalité $V_{i}\left(F_{n}, p^{k}\right)=V_{j}\left(F_{n}, p^{k}\right)$ a lieu.

Nous montrons qu'il existe un entier explicite $n_{0}$ (dépendant de $F$ ) tel que pour $n \geq n_{0}$, on ait

$$
k\left(F_{n}, i, j\right)+h\left(F_{n}, i, j\right)=m\left(F_{n}\right)+v_{p}(i-j)
$$

Pour $n$ quelconque, on a seulement l'inégalité $k(F, i, j)+h(F, i, j) \geq m(F)+v_{p}(i-j)$. Les techniques employées sont une adaptation de celles de $[\mathbf{G}]$ à la situation considérée ici. Bien sûr on retrouve les résultats de $[\mathbf{G}]$ mentionnés plus haut.

Les noyaux de Tate généralisés $\bmod p^{k}$ apparaissent naturellement lorsqu'on s'intéresse à certains problèmes de capitulation (cf par exemple $[\mathbf{K M}]$ ou [V1]). Dans le dernier paragraphe, nous travaillons à affaiblir l'hypothèse selon laquelle $i$ et $j$ doivent être $F$-bons. On complète ainsi la preuve du théorème 4.9 de [V1] en affaiblissant l'hypothèse de Leopoldt, ce qui rend le résultat applicable en pratique (on peut supposer Leopoldt seulement pour le corps de base, voir loc. cit. rem. 4.13)

\section{Notations}

$F$ un corps de nombres.

$p$ un nombre premier impair.

$G_{F}$ le groupe de Galois absolu de $F$.

$G_{S}=G_{S}(F)$ le groupe de Galois de l'extension $(p)$-ramifiée maximale de $F$.

$F_{\infty}=\cup F_{n}$ la $\mathbb{Z}_{p^{-}}$-extension cyclotomique de $F$.

$\Gamma=\operatorname{Gal}\left(F_{\infty} / F\right)$.

$\Lambda=\mathbb{Z}_{p}[[\Gamma]]$ l'algèbre d'Iwasawa.

$m(F)=\max \left\{m, \mu_{p^{m}} \subset F\left(\mu_{p}\right)\right\}$.

$A^{\prime}(F)=\mathcal{C} l^{\prime}(F) \otimes \mathbb{Z}_{p}$ la $p$-partie du groupe des $(p)$-classes de $F$.

$X^{\prime}=\lim A^{\prime}\left(F_{n}\right)$.

$H^{q}(F, \overleftarrow{\bullet})$ la cohomologie continue de $G_{F}$.

$H_{S}^{q}(F, \bullet)=H^{q}\left(G_{S}(F), \bullet\right)$ la cohomologie continue de $G_{S}(F)$.

$H_{I w}^{q}\left(F_{\infty}, \mathbb{Z}_{p}(i)\right)=\lim _{\longleftarrow} H_{S}^{q}\left(F_{n}, \mathbb{Z}_{p}(i)\right)$.

$X^{(i)}\left(F_{\infty}\right)=H_{I w}^{2}\left(F_{\infty}, \mathbb{Z}_{p}(i)\right)$.

$\mathcal{X}^{(-i)}(F)=H_{S}^{1}\left(F, \mathbb{Q}_{p} / \mathbb{Z}_{p}(i)\right)^{\vee}$ où $\bullet \vee$ désigne la dualité de Pontryagin.

$\mathcal{X}^{(-i)}\left(F_{\infty}\right)=\lim \mathcal{X}^{(-i)}\left(F_{n}\right)$.

$V_{i}\left(F, p^{k}\right)=\operatorname{Im}\left(H_{S}^{1}\left(F, \mathbb{Z}_{p}(i)\right) \rightarrow H_{S}^{1}\left(F, \mathbb{Z} / p^{k}(i)\right)\right)$, le $i^{\text {ème }}$ noyau de Tate $\bmod p^{k}$, si $i \neq 1$.

$V_{i}=D^{(i)}(F) / p$, voir cor. 1.5 pour $D^{(i)}(F)$ et $D^{(i)}\left(F, p^{k}\right)$.

$V_{i}\left(E / F, p^{k}\right)=\operatorname{Im}\left(H_{S}^{1}\left(E, \mathbb{Z}_{p}(i)\right)^{G(E / F)} \rightarrow H_{S}^{1}\left(E, \mathbb{Z} / p^{k}(i)\right)\right)$ la partie $F$-rationnelle du $i^{\text {ème }}$ noyau de Tate $\bmod p^{k}$.

$\exp (M)$ l'exposant du groupe abélien fini $M$.

$M^{\text {div }}$ le sous-groupe divisible maximal du groupe abélien $M$. 
$M^{0}$ le sous module fini maximal du $\Lambda$-module noethérien $M$.

$h=\exp \left(X^{\prime}\left(F_{\infty}\right)^{0}\right)$ l'exposant du $p$-groupe fini $X^{\prime}\left(F_{\infty}\right)^{0}$.

$h(F, i, j)=\min \left(\exp \left(X^{(i)}\left(F_{\infty}\right)^{\Gamma}\right), \exp \left(X^{(j)}\left(F_{\infty}\right)^{\Gamma}\right)\right)$.

$k(F, i, j)=\max \left\{k \leq m(F)+v_{p}(i-j), V_{i}\left(F, p^{k}\right)=V_{j}\left(F, p^{k}\right)\right\}$.

\section{Définition des noyaux de Tate généralisés}

Soit $F$ un corps de nombres et supposons momentanément qu'il contient $\zeta_{p}$, une racine primitive $p^{\text {ième }}$ de l'unité. Notons $\{.,$.$\} le symbole de Steinberg. Le noyau de Tate est habituellement défini$ comme le sous-groupe suivant $D^{(2)}(F)$ de $F^{\times}$:

$$
D^{(2)}(F):=\left\{x \in F^{\times},\left\{x, \zeta_{p}\right\}=0\right\}
$$

et comme il contient $F^{\times p}$, l'étude de $D^{(2)}(F)$ est strictement équivalente à celle de sa réduction $\bmod p: V_{2}(F, p)=D^{(2)}(F) / p \subset F^{\times} / F^{\times p}$ que l'on qualifiera de noyau de Tate $\bmod p$. Nous proposons ici, pour $F$ quelconque (ne contenant pas nécessairement $\mu_{p}$ ) et $i \neq 1$, une définition cohomologique directe et générale des noyaux de Tate $\bmod p^{k}$. Pour $F$ contenant $\mu_{p}$ et $k=1$, nous retrouverons les groupes $D^{(i)}(F) / p$, où $D^{(i)}(F)$ est la généralisation naturelle de $D^{(2)}(F)$ (voir cor. 1.5). Notons $H^{q}(F, \bullet)$ (resp. $H_{S}^{q}(F, \bullet)$ ) la cohomologie continue du groupe de Galois absolu $G_{F}$ de $F$ (resp. du groupe de Galois $G_{S}$ de l'extension $(p)$-ramifiée maximale de $F$ ) comme définie dans [T].

Définition 1.1. - Soit $F$ un corps de nombres quelconque, $i \in \mathbb{Z}, i \neq 1$. On note $V_{i}\left(F, p^{k}\right)$ l'image du morphisme naturel

$$
H_{S}^{1}\left(F, \mathbb{Z}_{p}(i)\right) \rightarrow H_{S}^{1}\left(F, \mathbb{Z} / p^{k}(i)\right)
$$

On l'appelle le ième noyau de Tate $\bmod p^{k}$.

Remarque 1.2. - Le cas $i=1$ demanderait un traitement particulier. Pour éviter les complications inutiles, nous l'éviterons systématiquement dans cet article, et nous y reviendrons dans [V2], où une étude différente sera proposée.

L'objet de ce travail est la comparaison - lorsqu'elle a un sens - des différents noyaux de Tate $\bmod p^{k}$. Pour que cette comparaison soit possible, il faut pouvoir identifier les groupes $H_{S}^{1}\left(F, \mathbb{Z} / p^{k}(i)\right)$ et $H_{S}^{1}\left(F, \mathbb{Z} / p^{k}(j)\right)$; nous demanderons donc que l'image de $G_{S}$ par la puissance $(i-j)^{\text {ième }}$ du caractère cyclotomique $\bmod p^{k}$ (ie. à valeurs dans $\left.\left(\mathbb{Z} / p^{k}\right)^{\times}\right)$soit triviale, ce qui revient à $i \equiv j \bmod \left[F\left(\mu_{p}\right): F\right]$ et

$$
k \leq m(F)+v_{p}(i-j)
$$

où $m(F)=\max \left\{m, \mu_{p^{m}} \subset F\left(\mu_{p}\right)\right\}$. Lorsque c'est le cas, on identifiera $V_{i}\left(F, p^{k}\right)$ à un sous-groupe de $H_{S}^{1}\left(F, \mathbb{Z} / p^{k}(j)\right)$ et l'on dira que $V_{i}\left(F, p^{k}\right)$ et $V_{j}\left(F, p^{k}\right)$ sont comparables.

Remarque 1.3. - La définition ci-dessus permet de changer $k$ facilement : comme le diagramme à lignes exactes suivant commute

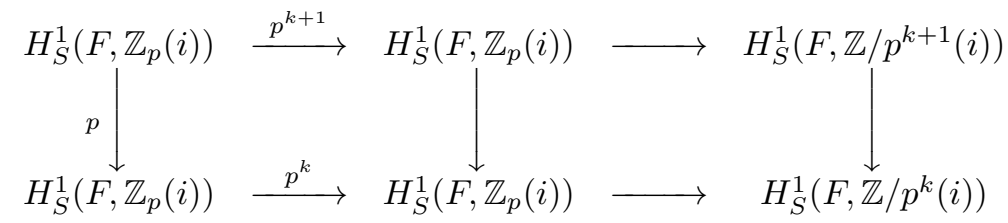

la dernière flèche verticale permet d'identifier naturellement $V_{i}\left(F, p^{k}\right)$ avec $V_{i}\left(F, p^{k+1}\right) / p^{k}$.

Remarque 1.4. - L'inflation de $G_{S} \grave{a} G_{F}$ permet de voir $H_{S}^{1}\left(F, \mathbb{Z} / p^{k}(i)\right)$ comme un sous-groupe de $H^{1}\left(F, \mathbb{Z} / p^{k}(i)\right)$. Comme on suppose $i \neq 1$, l'inflation produit aussi un isomorphisme canonique $H_{S}^{1}\left(F, \mathbb{Z}_{p}(i)\right)=H^{1}\left(F, \mathbb{Z}_{p}(i)\right)$ (c'est une conséquence directe de la suite exacte de localisation en cohomologie étale, cf $[\mathbf{S}])$. On peut donc voir $V_{i}\left(F, p^{k}\right)$ comme le sous-groupe $H^{1}\left(F, \mathbb{Z}_{p}(i)\right) / p^{k}$ de $H^{1}\left(F, \mathbb{Z} / p^{k}(i)\right)$. 
Proposition 1.1. - Soient $i \equiv j \bmod \left[F\left(\mu_{p}\right): F\right], k \leq m(F)+v_{p}(i-j)$ et notons $\delta^{(i-j)} l e$ $G_{F}$-cobord associé à la suite exacte

$$
0 \rightarrow \mathbb{Z}_{p}(i-j) \stackrel{p^{k}}{\longrightarrow} \mathbb{Z}_{p}(i-j) \longrightarrow \mathbb{Z} / p^{k}(i-j) \rightarrow 0
$$

Alors le sous-groupe $H^{1}\left(F, \mathbb{Z}_{p}(i)\right) / p^{k} \cap H^{1}\left(F, \mathbb{Z}_{p}(j)\right) / p^{k}$ de $H^{1}\left(F, \mathbb{Z}_{p}(j)\right) / p^{k}$ est l'orthogonal de $\delta^{(i-j)}\left(\mathbb{Z} / p^{k}(i-j)\right)$ pour le cup-produit

$$
H^{1}\left(F, \mathbb{Z}_{p}(j)\right) / p^{k} \otimes H^{1}\left(F, \mathbb{Z}_{p}(i-j)\right)\left[p^{k}\right] \rightarrow H^{2}\left(F, \mathbb{Z}_{p}(i)\right)
$$

Preuve : La fonctorialité du cup produit par rapport au décalage donne un diagramme commutatif

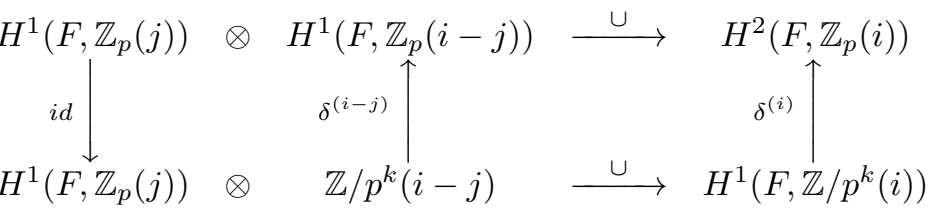

puis un autre :

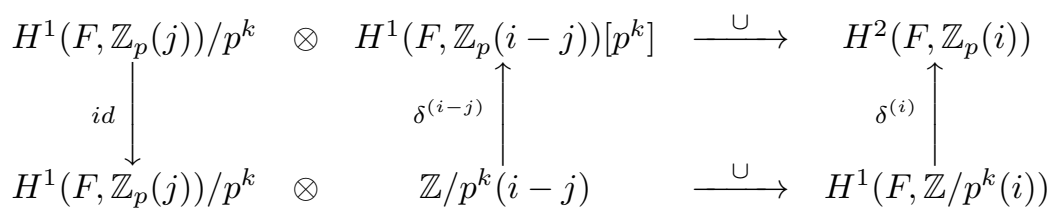

Maintenant, le cup produit de la ligne inférieure est simplement l'inclusion de groupes

$$
H^{1}\left(F, \mathbb{Z}_{p}(j)\right) / p^{k} \subset H^{1}\left(F, \mathbb{Z} / p^{k}(i)\right) .
$$

On voit donc sur ce diagramme que l'orthogonal de $\delta^{(i-j)}\left(\mathbb{Z} / p^{k}(i-j)\right)$, vu comme sous-groupe de $H^{1}\left(F, \mathbb{Z}_{p}(j)\right) / p^{k} \subset H^{1}\left(F, \mathbb{Z} / p^{k}(i)\right)$, est l'intersection de $H^{1}\left(F, \mathbb{Z}_{p}(j)\right) / p^{k}$ avec le noyau de l'homomorphisme de Bockstein

$$
\delta^{(i)}: H^{1}\left(F, \mathbb{Z} / p^{k}(i)\right) \rightarrow H^{2}\left(F, \mathbb{Z}_{p}(i)\right)
$$

Mais le noyau en question est $H^{1}\left(F, \mathbb{Z}_{p}(i)\right) / p^{k}$ et cela termine la preuve.

Notons $\delta_{1}$ l'application de Kummer, c'est-à-dire le morphisme de connexion associé à la suite exacte

$$
0 \rightarrow \mathbb{Z}_{p}(1) \longrightarrow \lim _{\longleftarrow} \bar{F}^{\times} \longrightarrow \bar{F}^{\times} \rightarrow 0
$$

Corollaire 1.5. - Soit $i \equiv 1 \bmod \left[F\left(\mu_{p}\right): F\right], i \neq 1, k \leq m(F)+v_{p}(i-1)$. Notons $D^{(i)}\left(F, p^{k}\right)$ le sous-groupe de $F^{\times}$orthogonal $\grave{a} \mu_{p}^{\otimes i-1}$ pour le symbole

$$
F^{\times} \otimes \mu_{p^{k}}^{\otimes i-1} \stackrel{(., .)}{\longrightarrow} H^{2}\left(F, \mathbb{Z}_{p}(i)\right)
$$

défini par $(x, y)=\delta x \cup \delta_{1} y$. Alors $V_{i}\left(F, p^{k}\right)$, vu comme sous-groupe de $F^{\times} / F^{\times p}$, s'identifie à $D^{(i)}\left(F, p^{k}\right) / p^{k}$. En particulier, $V_{i}(F, p)$ s'identifie avec $V_{i}:=D^{(i)}(F) / p$, où $D^{(i)}(F):=D^{(i)}(F, p)$ est par définition le noyau de Tate généralisé de $[\mathbf{A M}],[\mathbf{K M}]$ et $[\mathbf{H}]$.

Preuve : Compte tenu de la remarque 1.4, il suffit d'appliquer la proposition précédente avec $j=1$, en remarquant que

$$
H^{1}\left(F, \mathbb{Z} / p^{k}(1)\right)=F^{\times} / F^{\times p^{k}}=H^{1}\left(F, \mathbb{Z}_{p}(1)\right) / p^{k}
$$

Ce corollaire justifie l'appellation "noyau de Tate $\bmod p^{k}$ ".

Définition 1.6. - Soit $X^{(i)}\left(F_{\infty}\right)=H_{I w}^{2}\left(F_{\infty}, \mathbb{Z}_{p}(i)\right)$, et $\delta_{i}=r g_{\mathbb{Z}_{p}} X^{(i)}\left(F_{\infty}\right)^{\Gamma}$. On dit que $i$ est $F$-bon si $\delta_{i}=0$.

Remarque 1.7. - Puisque $X^{(i)}\left(F_{\infty}\right)_{\Gamma}=H_{S}^{2}\left(F, \mathbb{Z}_{p}(i)\right)$ (cela tient à $c d_{p} G_{S} \leq 2$, voir [V3] pour plus de détails), $\delta_{i}$ est le $\mathbb{Z}_{p}$-rang de $H_{S}^{2}\left(F, \mathbb{Z}_{p}(i)\right)$. C. Soulé a montré que tout $i \geq 2$ est $F$-bon $[\mathbf{S}]$. Par ailleurs, 0 l'est si et seulement si $F$ vérifie la conjecture de Leopoldt en $p$. 
Proposition 1.2. - Notons s le nombre de $(p)$-places de $F$, et $\epsilon_{i}=1$ si $i \equiv 1 \bmod \left[F\left(\mu_{p}\right): F\right]$ ou $i=0, \epsilon_{i}=0$ sinon. Le p-rang de $V_{i}\left(F, p^{k}\right)$ est alors donné par $r_{2}+\delta_{i}+\epsilon_{i}$ si $i$ est pair, $r_{1}+r_{2}+\delta_{i}+\epsilon_{i}$ si est impair.

Preuve : Il s'agit d'un résultat facile, dont nous donnons rapidement les éléments de la preuve. On renvoie à $[\mathbf{V 3}]$ pour plus de détails. Pour simplifier, on ne traite que le cas $i \neq 0$. On a alors $H_{S}^{1}\left(F, \mathbb{Z}_{p}(i)\right)[p]=\mathbb{Z} / p(i)^{G_{S}}$, et le $p$-rang annoncé est obtenu en ajoutant $\epsilon_{i}$ au $\mathbb{Z}_{p}$-rang de $H_{S}^{1}\left(F, \mathbb{Z}_{p}(i)\right)$. Pour $i \geq 2$, ce dernier est bien connu : il résulte, via $[\mathbf{S}]$, d'un résultat de Borel concernant les groupes de $K$-théorie algébrique. Pour $i \neq 0, i<2$, on se ramène au cas $i \geq 2$ de la façon suivante : on a $\mathcal{X}^{(-i)}(F)=\mathcal{X}^{(-i)}\left(F_{\infty}\right)_{\Gamma}$ où $F_{\infty} / F$ est la $\mathbb{Z}_{p}$-extension cyclotomique de $F$ et $\Gamma$ son groupe de Galois. Il est facile d'en déduire $r g_{\mathbb{Z}_{p}} \mathcal{X}^{(-i)}(F)=r g_{\Lambda} \mathcal{X}^{(-i)}\left(F_{\infty}\right)+r g_{\mathbb{Z}_{p}} t_{\Lambda} \mathcal{X}^{(-i)}\left(F_{\infty}\right)_{\Gamma}$. Maintenant le $\Lambda$-rang de $\mathcal{X}^{(-i)}\left(F_{\infty}\right)$ est le même que celui de $\mathcal{X}^{(-j)}\left(F_{\infty}\right)$ où $j \equiv i \bmod \left[F\left(\mu_{p}\right): F\right]$, $j \geq 2$; et ce dernier se déduit facilement des formules de Borel pour tous les $F_{n}$. Par ailleurs, on sait que $t_{\Lambda} \mathcal{X}^{(-i)}\left(F_{\infty}\right)$ est pseudo-isomorphe au module $X^{(i)}\left(F_{\infty}\right)$ dont l'action de $\Gamma$ aurait été inversée. Le résultat s'en déduit facilement.

\section{Comparaison $\bmod p^{k}$}

On fixe $i \equiv j \bmod \left[F\left(\mu_{p}\right): F\right]$, et on suppose toujours que $V_{i}\left(F, p^{k}\right)$ et $V_{j}\left(F, p^{k}\right)$ sont comparables, ie. $k \leq m(F)+v_{p}(i-j)$.

Commençons par rappeler les notations évoquées dans l'introduction :

Définition 2.1. - Soit $F_{\infty} / F$ la $\mathbb{Z}_{p}$-extension cyclotomique de $F=F_{0}, \Gamma$ son groupe de Galois, et $X^{(i)}\left(F_{\infty}\right)=H_{I w}^{2}\left(F, \mathbb{Z}_{p}(i)\right)$ le module d'Iwasawa habituel (cf Notations). Pour $i, j F_{n}$-bons (cf def 1.6), on adopte les notations suivantes :

- $h$ est l'exposant du sous-module fini maximal (aussi appelé noyau de capitulation) $X^{(i)}\left(F_{\infty}\right)^{0}$ de $X^{(i)}\left(F_{\infty}\right)$. Notons que si $i \equiv 1 \bmod \left[F\left(\mu_{p}\right): F\right]$, les groupes $X^{(i)}\left(F_{\infty}\right)^{0}$ et $X^{\prime}\left(F_{\infty}\right)^{0}$ sont égaux.

- $h(F, i, j)$ est le plus petit des exposants des groupes $X^{(i)}\left(F_{\infty}\right)^{\Gamma}$ et $X^{(j)}\left(F_{\infty}\right)^{\Gamma}$ (ces groupes sont finis car $i$ est $j$ sont $F_{n}$-bons).

- $k(F, i, j)=\max \left\{k \leq m(F)+v_{p}(i-j), V_{i}\left(F, p^{k}\right)=V_{j}\left(F, p^{k}\right)\right\}$ est la précision maximale d'égalité des $i^{\text {ième }}$ et jième noyaux de Tate.

- $n_{0}$ est le plus petit étage $n$ tels que l'un des deux modules $X^{(i)}\left(F_{\infty}\right)^{0}, X^{(j)}\left(F_{\infty}\right)^{0}$ soit fixé par $\Gamma_{n}$.

L'objet de ce paragraphe est la preuve du résultat principal :

Théorème 2.2. - Soient $i, j$ deux entiers $F_{n}$-bons, congrus $\bmod \left[F\left(\mu_{p}\right): F\right]$. Alors on a l'égalité suivante

pour tout $n \geq n_{0}$. De plus l'inégalité

$$
k\left(F_{n}, i, j\right)+h\left(F_{n}, i, j\right)=m\left(F_{n}\right)+v_{p}(i-j)
$$

$$
k\left(F_{n}, i, j\right)+h\left(F_{n}, i, j\right) \geq m\left(F_{n}\right)+v_{p}(i-j)
$$

est vraie pour tout $n \geq 0$.

Avant de passer à la preuve, notons tout de suite que les résultats de $[\mathbf{G}]$ mentionnés dans l'introduction sont contenus dans ce théorème. En effet, notant $V_{i}=V_{i}(F, p)$, on retrouve :

Proposition 2.1. - ([G] §6) Si F vérifie la conjecture de Leopoldt et contient $\mu_{p}$, alors

(i) Si $m(F) \geq h+1$, alors $V_{0}=V_{2}$.

(ii) Si $X^{\prime}\left(F_{\infty}\right)^{0}$ est cyclique d'ordre $p$ et si $m(F)=1$, alors $V_{0} \neq V_{2}$.

Preuve : 0 est $F$-bon puique $F$ vérifie la conjecture de Leopoldt; et 2 l'est toujours (cf rem. 1.7). (i) Comme $h(F, 2,0) \leq h$ et $v_{p}(2-0)=0$, l'inégalité du théorème 2.2 donne

$$
k(F, 2,0) \geq m(F)-h(F, 2,0) \geq m(F)-h \geq 1
$$


si bien que $V_{0}=V_{2}$ par définition de $k(F, 2,0)$.

(ii) Si $h=1$, alors on a automatiquement $n_{0}=0$ et $h(F, 2,0)=h=1$. L'égalité du théorème 2.2 donne donc

ie. $V_{2} \neq V_{0}$.

$$
k(F, 2,0)=m(F)-h=0
$$

Les résultats de $[\mathbf{A M}]$ et $[\mathbf{H}]$ ayant trait à la comparaison des noyaux de Tate généralisés pour $i \neq 1$ se déduisent de l'inégalité de 2.2 de la même manière.

Pour prouver 2.2, nous aurons besoin du lemme suivant :

Lemme 2.3. - Soit $i \in \mathbb{Z}$; si $F$ contient $\mu_{p^{k}}$, alors les sous-groupes $V_{i}\left(F, p^{k}\right)$ et $V_{j}\left(F, p^{k}\right)$ de $H_{S}^{1}\left(F, \mathbb{Z} / p^{k}(j)\right)$ sont les images réciproques respectives de $\left(H_{S}^{1}\left(F_{\infty}, \mathbb{Q}_{p} / \mathbb{Z}_{p}(i)\right)^{\Gamma}\right)^{d i v}\left[p^{k}\right]$ et de $\left(H_{S}^{1}\left(F_{\infty}, \mathbb{Q}_{p} / \mathbb{Z}_{p}(j)\right)^{\Gamma}\right)^{d i v}\left[p^{k}\right]$ vus comme sous-groupe de $H_{S}^{1}\left(F_{\infty}, \mathbb{Q}_{p} / \mathbb{Z}_{p}(j)\right)$ (comme groupe c'est aussi $\left.H_{S}^{1}\left(F_{\infty}, \mathbb{Q}_{p} / \mathbb{Z}_{p}(i)\right)\right)$, par l'application naturelle

$$
\phi_{j}: H_{S}^{1}\left(F, \mathbb{Z} / p^{k}(j)\right) \rightarrow H_{S}^{1}\left(F_{\infty}, \mathbb{Q}_{p} / \mathbb{Z}_{p}(j)\right)
$$

Preuve : Considérons le diagramme commutatif à lignes exactes suivant :

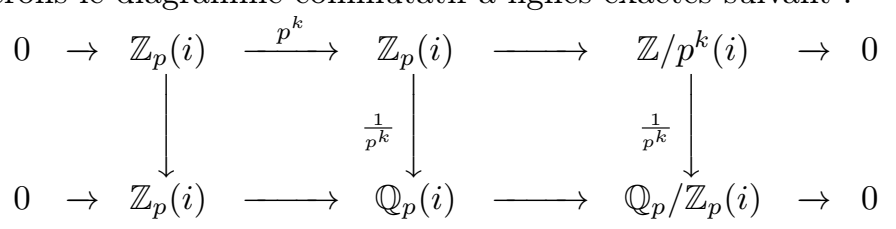

En prenant la $G_{S}$-cohomologie, on obtient le diagramme commutatif à lignes et colonnes exactes :

$$
\begin{array}{ccccc}
Z_{i} & & Z_{i} & \hookrightarrow & 0 \\
\downarrow & & \downarrow & & \downarrow \\
H_{S}^{1}\left(F, \mathbb{Z}_{p}(i)\right) / p^{k} & \hookrightarrow & H_{S}^{1}\left(F, \mathbb{Z} / p^{k}(i)\right) & \rightarrow & H_{S}^{2}\left(F, \mathbb{Z}_{p}(i)\right)\left[p^{k}\right] \\
\downarrow & & f_{i} \downarrow & & \downarrow \\
H_{S}^{1}\left(F, \mathbb{Z}_{p}(i)\right) \otimes \mathbb{Q}_{p} / \mathbb{Z}_{p}\left[p^{k}\right] & \stackrel{g_{i}}{\hookrightarrow} & H_{S}^{1}\left(F, \mathbb{Q}_{p} / \mathbb{Z}_{p}(i)\right)\left[p^{k}\right] & \rightarrow & H_{S}^{2}\left(F, \mathbb{Z}_{p}(i)\right)\left[p^{k}\right] \\
\downarrow & & \downarrow & & \downarrow \\
0 & & 0 & & 0
\end{array}
$$

où $Z_{i}$ désigne $H_{S}^{0}\left(F, \mathbb{Z} / p^{k}(i)\right)$ si $i \neq 0$ et 0 si $i=0$. On voit sur ce diagramme que $V_{i}\left(F, p^{k}\right)=$ $f_{i}^{-1}\left(\operatorname{Im} g_{i}\right)=H_{S}^{1}\left(F, \mathbb{Q}_{p} / \mathbb{Z}_{p}(i)\right)^{d i v}\left[p^{k}\right]$. Comme $H^{1}\left(\Gamma, \mathbb{Q}_{p} / \mathbb{Z}_{p}(i)\right)$ est divisible, on obtient par inflation-restriction la suite exacte suivante :

$$
H^{1}\left(\Gamma, \mathbb{Q}_{p} / \mathbb{Z}_{p}(i)\right)\left[p^{k}\right] \quad \hookrightarrow \quad H_{S}^{1}\left(F, \mathbb{Q}_{p} / \mathbb{Z}_{p}(i)\right)^{d i v}\left[p^{k}\right] \quad \rightarrow \quad\left(H_{S}^{1}\left(F_{\infty}, \mathbb{Q}_{p} / \mathbb{Z}_{p}(i)\right)^{\Gamma}\right)^{d i v}\left[p^{k}\right]
$$

Finalement, $V_{i}\left(F, p^{k}\right)$ est l'image réciproque de $H_{S}^{1}\left(F, \mathbb{Q}_{p} / \mathbb{Z}_{p}(i)\right)^{d i v}\left[p^{k}\right]$ par l'application composée

$$
\phi_{i}: H_{S}^{1}\left(F, \mathbb{Z} / p^{k}(i)\right) \stackrel{f_{i}}{\rightarrow} H_{S}^{1}\left(F, \mathbb{Q}_{p} / \mathbb{Z}_{p}(i)\right) \stackrel{r^{2} s_{F}^{F}}{\rightarrow} H^{1}\left(F_{\infty}, \mathbb{Q}_{p} / \mathbb{Z}_{p}(i)\right)
$$

Maintenant, les applications $\phi_{i}$ et $\phi_{j}$ coïncident en tant que morphismes de groupes, comme on peut s'en convaincre en observant le carré commutatif suivant :

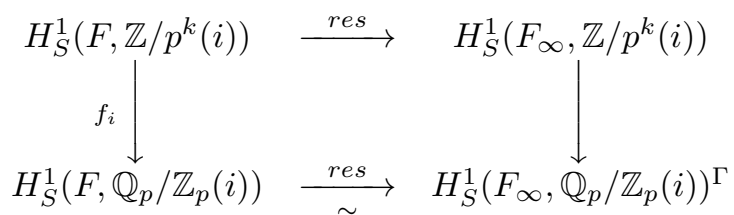

Preuve de 2.2: Quitte à changer $F$, on peut toujours supposer $F_{n}=F$. Cela revient à traduire la condition supplémentaire " $n \geq n_{0}$ " par "l'un des deux modules $X^{(i)}\left(F_{\infty}\right)^{0}, X^{(j)}\left(F_{\infty}\right)^{0}$ est fixé par $\Gamma$ ". Il s'agit de montrer que

$$
k \leq m(F)+v_{p}(i-j)-h(F, i, j) \Rightarrow V_{i}\left(F, p^{k}\right)=V_{j}\left(F, p^{k}\right)
$$

et que sous la condition supplémentaire, on a en fait l'équivalence. 
D'après le lemme 2.3, $V_{i}\left(F, p^{k}\right)$ et $\left.V_{j}\left(F, p^{k}\right)\right)$ sont égaux si et seulement si les sous-groupes $\left(H_{S}^{1}\left(F_{\infty}, \mathbb{Q}_{p} / \mathbb{Z}_{p}(i)\right)^{\Gamma}\right)^{d i v}\left[p^{k}\right]$ et $\left(H_{S}^{1}\left(F_{\infty}, \mathbb{Q}_{p} / \mathbb{Z}_{p}(j)\right)^{\Gamma}\right)^{d i v}\left[p^{k}\right]$ de $H^{1}\left(F_{\infty}, \mathbb{Z} / p^{k}(j)\right)^{\Gamma}$ (lui-même sousgroupe de $\left.H_{S}^{1}\left(F_{\infty}, \mathbb{Q}_{p} / \mathbb{Z}_{p}(j)\right)\right)$ sont égaux. En passant aux orthogonaux via l'accouplement parfait suivant (voir Notations pour $\mathcal{X}^{(-j)}$ )

$$
H_{S}^{1}\left(F_{\infty}, \mathbb{Z}_{p} / p^{k}(j)\right)^{\Gamma} \otimes \mathcal{X}^{(-j)}\left(F_{\infty}\right)_{\Gamma} / p^{k} \rightarrow \mathbb{Z} / p^{k}
$$

on obtient l'équivalence

$$
V_{i}\left(F, p^{k}\right)=V_{j}\left(F, p^{k}\right) \Leftrightarrow\left(t_{\mathbb{Z}_{p}}\left(\mathcal{X}^{(-i)}\left(F_{\infty}\right)_{\Gamma}\right)\right) / p^{k}=\left(t_{\mathbb{Z}_{p}}\left(\mathcal{X}^{(-j)}\left(F_{\infty}\right)_{\Gamma}\right)\right) / p^{k}
$$

où l'on regarde $\left(t_{\mathbb{Z}_{p}}\left(\mathcal{X}^{(-i)}\left(F_{\infty}\right)_{\Gamma}\right)\right) / p^{k}$ et $\left(t_{\mathbb{Z}_{p}}\left(\mathcal{X}^{(-j)}\left(F_{\infty}\right)_{\Gamma}\right)\right) / p^{k}$ comme des sous-espaces de

$$
\mathcal{X}^{(-j)}\left(F_{\infty}\right)_{\Gamma} / p^{k}=\mathcal{X}^{(-j)}\left(F_{\infty}\right)_{\Gamma} / p^{k} .
$$

Maintenant $i$ et $j$ sont supposés $F$-bons, $\left(t_{\Lambda} \mathcal{X}^{(-i)}\left(F_{\infty}\right)\right)_{\Gamma}$ et $\left(t_{\Lambda} \mathcal{X}^{(-j)}\left(F_{\infty}\right)\right)_{\Gamma}$ sont donc de $\mathbb{Z}_{p^{-}}$ torsion (cf [V3], Cor. 1.2.41), si bien que

$$
\left(t_{\Lambda} \mathcal{X}^{(-j)}\left(F_{\infty}\right)\right)_{\Gamma} / p^{k} \subset\left(t_{\mathbb{Z}_{p}}\left(\mathcal{X}^{(-i)}\left(F_{\infty}\right)_{\Gamma}\right)\right) / p^{k} \cap\left(t_{\mathbb{Z}_{p}}\left(\mathcal{X}^{(-j)}\left(F_{\infty}\right)_{\Gamma}\right)\right) / p^{k}
$$

L'égalité en question équivaut donc à la suivante, en tant que sous-groupes de $\left(f_{\Lambda} \mathcal{X}^{(-i)}\left(F_{\infty}\right)\right)_{\Gamma} / p^{k}$ :

$$
\left(t_{\mathbb{Z}_{p}}\left(f_{\Lambda} \mathcal{X}^{(-i)}\left(F_{\infty}\right)\right)_{\Gamma}\right) / p^{k}=\left(t_{\mathbb{Z}_{p}}\left(f_{\Lambda} \mathcal{X}^{-j)}\left(F_{\infty}\right)\right)_{\Gamma}\right) / p^{k}
$$

Il est bien connu (Voir par ex. [J] cor. 6.5 b, ou [V3], rem. 3.2.27) que le $\Lambda$-module $\left(X^{(i)}\left(F_{\infty}\right)^{0}\right)^{\vee}$ est isomorphe au conoyau de l'application canonique

$$
f_{\Lambda} \mathcal{X}^{(-i)}\left(F_{\infty}\right) \rightarrow \operatorname{Hom}_{\Lambda}\left(\operatorname{Hom}_{\Lambda}\left(f_{\Lambda} \mathcal{X}^{(-i)}\left(F_{\infty}\right), \Lambda\right), \Lambda\right)
$$

La preuve de la proposition 2.2 se réduit donc au lemme algébrique suivant, en prenant $M=$ $f_{\Lambda} \mathcal{X}^{(-i)}\left(F_{\infty}\right), e=i-j$ et $h^{\prime}=h(F, i, j)$.

Lemme 2.4. - Soit $M$ un $\Lambda$-module noethérien, sans torsion et soit $e \in \mathbb{Z}$. Soit $H$ le conoyau de l'application naturelle $M \rightarrow \operatorname{Hom}_{\Lambda}\left(\operatorname{Hom}_{\Lambda}(M, \Lambda), \Lambda\right)$. Notons encore

$$
h^{\prime}=\min \left(v_{p}\left(\exp \left(H^{\Gamma}\right)\right), v_{p}\left(\exp \left(H(e)^{\Gamma}\right)\right)\right)
$$

et

$$
n=v_{p}\left(\left(\mathbb{Z}_{p} \times: \kappa(\Gamma)\right)\right)+1=m(F)
$$

$\kappa$ étant le caractère cyclotomique. Si $v_{p}(e) \geq h^{\prime}-n+k$, alors les sous-groupes $\left(t_{\mathbb{Z}_{p}} M_{\Gamma}\right) / p^{k}$ et $\left(t_{\mathbb{Z}_{p}} M(e)_{\Gamma}\right) / p^{k}$ de $M_{\Gamma} / p^{k}=M(e)_{\Gamma} / p^{k}$ sont égaux.

Si l'on suppose de plus que l'un des deux $\Lambda$-modules $H, H(e)$ est fixé par $\Gamma$, alors on a l'équivalence

$$
\left(t_{\mathbb{Z}_{p}} M_{\Gamma}\right) / p^{k}=\left(t_{\mathbb{Z}_{p}} M(e)_{\Gamma}\right) / p^{k} \Leftrightarrow v_{p}(e) \geq h^{\prime}-n+k
$$

Preuve de 2.4, sens direct : Nous utiliserons le lemme suivant :

Lemme 2.5. - Soit $Z$ un $\mathbb{Z}_{p}$-module noethérien, $h^{\prime}$ et $k$ deux entiers naturels, alors l'image de l'application naturelle $Z / p^{h^{\prime}+k}\left[p^{h^{\prime}}\right] \rightarrow f_{\mathbb{Z}_{p}} Z / p^{k}$ est triviale.

Preuve : C'est trivial si l'on décompose $Z=t_{\mathbb{Z}_{p}} Z \oplus f_{\mathbb{Z}_{p}} Z$.

Supposons $n+v_{p}(e) \geq h^{\prime}+k$, on peut alors faire les identifications suivantes : $M_{\Gamma} / p^{h^{\prime}+k}=$ $M(e)_{\Gamma} / p^{h^{\prime}+k}$ et $M_{\Gamma} / p^{k}=M(e)_{\Gamma} / p^{k}$. Il s'agit de montrer que $t_{\mathbb{Z}_{p}} M_{\Gamma} / p^{k}$ et $t_{\mathbb{Z}_{p}} M(e)_{\Gamma} / p^{k}(-e)$, vus comme sous-modules de $M_{\Gamma} / p^{k}$, sont égaux. D'abord ils ont même ordre puisque

$$
\#\left(f_{\mathbb{Z}_{p}} M_{\Gamma} / p^{k}\right)=\#\left(f_{\mathbb{Z}_{p}} M(e)_{\Gamma} / p^{k}\right)=p^{k r g_{\Lambda} M}
$$

Ensuite le lemme 2.5 appliqué à $Z=M_{\Gamma}$ et $Z=M(e)_{\Gamma}$ montre que tous deux contiennent l'image de l'application naturelle $M_{\Gamma} / p^{h^{\prime}+k}\left[p^{h^{\prime}}\right] \rightarrow M_{\Gamma} / p^{k}$. On peut supposer sans perte de généralité que $h^{\prime}=v_{p}\left(\exp \left(H^{\Gamma}\right)\right)$. Dans ce cas $v_{p}\left(\exp \left(t_{\mathbb{Z}_{p}}\left(M_{\Gamma}\right)\right)\right)=h^{\prime}$ et $M_{\Gamma} / p^{h^{\prime}+k}\left[p^{h^{\prime}}\right]$ contient $t_{\mathbb{Z}_{p}} M_{\Gamma} / p^{h^{\prime}+k}$ et l'on en déduit que $t_{\mathbb{Z}_{p}} M(e)_{\Gamma}(-e)$ contient $t_{\mathbb{Z}_{p}} M_{\Gamma} / p^{k}$, ce qui termine la preuve puisqu'ils ont même ordre. 
Preuve de 2.4, suite : Passons à l'équivalence dans le cas où l'un des deux $\Lambda$-modules $H, H(e)$ est fixé par $\Gamma$. Nous utiliserons le lemme suivant :

Lemme 2.6. - On conserve les hypothèses et notations du lemme 2.4. Soit Z un $\Lambda$-module fini. Si $Z$ et $Z(e)$ sont fixés par $\Gamma$, c'est que $v_{p}(\exp (Z)) \leq v_{p}(e)+n$.

Preuve : C'est immédiat si l'on décompose $Z$ en $\Lambda$-modules cycliques (comme groupes).

Il s'agit de montrer que si $t_{\mathbb{Z}_{p}} M_{\Gamma} / p^{k}=t_{\mathbb{Z}_{p}} M(e)_{\Gamma} / p^{k}$, alors $v_{p}(e)+n \geq h^{\prime}+k$.

Comme dans [G], $\S 6$., on définit les $\Lambda$-modules $Y, Y_{e}, Z$ et $Z_{e}$ par l'exactitude des suites suivantes

$$
\begin{aligned}
& 0 \rightarrow Y \longrightarrow M \quad \longrightarrow \quad \longrightarrow f_{\mathbb{Z}_{p}} M_{\Gamma} / p^{k} \rightarrow 0 \\
& 0 \rightarrow Y_{e} \longrightarrow M(e) \quad \longrightarrow \quad f_{\mathbb{Z}_{p}} M(e)_{\Gamma} \rightarrow 0 \\
& 0 \rightarrow Y \longrightarrow \operatorname{Hom}_{\Lambda}\left(\operatorname{Hom}_{\Lambda}(M, \Lambda), \Lambda\right) \longrightarrow Z \quad \longrightarrow \quad \longrightarrow 0 \\
& 0 \rightarrow Y_{e} \longrightarrow \operatorname{Hom}_{\Lambda}\left(\operatorname{Hom}_{\Lambda}(M, \Lambda), \Lambda\right)(e) \longrightarrow Z_{e} \rightarrow 0
\end{aligned}
$$

Par hypothèse, on a $t_{\mathbb{Z}_{p}} M_{\Gamma} / p^{k}=t_{\mathbb{Z}_{p}} M(e)_{\Gamma} / p^{k}$, et donc $Y_{e}=Y(e)$ et $Z_{e}=Z(e)$.

Considérons alors le diagramme suivant :

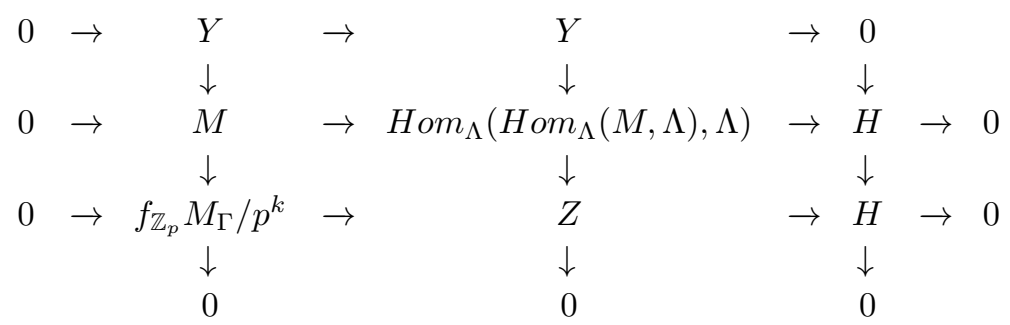

En prenant la $\Gamma$-cohomologie, on observe que le cobord

$$
H^{\Gamma} \rightarrow f_{\mathbb{Z}_{p}} M_{\Gamma} / p^{k}
$$

est nul. Cela résulte de la commutativité du diagramme

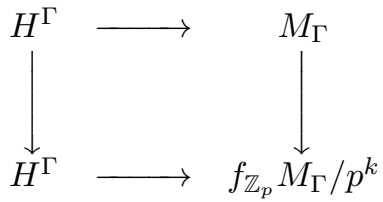

Ainsi, la suite

$$
0 \rightarrow f_{\mathbb{Z}_{p}} M_{\Gamma} / p^{k} \longrightarrow Z^{\Gamma} \longrightarrow H^{\Gamma} \rightarrow 0
$$

est exacte. De la même façon, on a une suite exacte

$$
0 \rightarrow f_{\mathbb{Z}_{p}} M(e)_{\Gamma} / p^{k} \longrightarrow Z(e)^{\Gamma} \longrightarrow H(e)^{\Gamma} \rightarrow 0
$$

Notons alors $Z^{\prime}$ l'image réciproque, dans $Z$, de $H^{\prime}=H^{\Gamma} \cap H(e)^{\Gamma}$. Les deux suites exactes cidessus montrent que $Z^{\prime}$ et $Z^{\prime}(e)$ sont fixés par $\Gamma$. Reste, pour appliquer le lemme 2.6, à déterminer l'exposant de $Z^{\prime}$. On peut supposer, sans perte de généralité, que $Z$ est fixé par $\Gamma$. La surjection $\Lambda^{r g_{\Lambda} M}=\operatorname{Hom}_{\Lambda}\left(\operatorname{Hom}_{\Lambda}(M, \Lambda), \Lambda\right) \rightarrow Z$ montre alors que $r g_{\mathbb{Z} / p} Z / p \leq r g_{\Lambda} M$. On en déduit que $r g_{\mathbb{Z} / p} Z^{\prime} / p \leq r g_{\Lambda} M$. La suite exacte tautologique

$$
0 \rightarrow f_{\mathbb{Z}_{p}} M_{\Gamma} / p^{k} \longrightarrow Z^{\prime} \longrightarrow H^{\prime} \rightarrow 0
$$

montre alors que $v_{p}\left(\exp \left(Z^{\prime}\right)\right)=k+v_{p}\left(\exp \left(H^{\prime}\right)\right)=k+h^{\prime}$ puisque $f_{\mathbb{Z}_{p}} M_{\Gamma} / p^{k}=\left(\mathbb{Z} / p^{k}\right)^{r g_{\Lambda} M}$. Grâce au lemme 2.6, on en conclut que $v_{p}(e)+n \geq k+h^{\prime}$, et cela termine la preuve. 
Remarque 2.7. - Si aucun des deux $\Lambda$-modules $H, H(e)$, n'est fixé par $\Gamma$ l'équivalence dans 2.4 n'est plus vraie (et donc a priori dans 2.2 non plus) : cela tient au fait qu'on ne contrôle plus le $p$-rang de $Z^{\prime}$. L'exemple suivant illustre bien la situation : soit $M$ l'idéal $\left((1+p)^{-1}(1+T)-1, p^{2}\right)$ de $\Lambda=\mathbb{Z}_{p}[[T]]$, et choisissons $n=e=k=1$. On a alors $H=\mathbb{Z} / p^{2}(1)$, si bien que ni $H$, ni $H(1)$ n'est fixé par $\Gamma$ et que $h^{\prime}=1$. On vérifie facilement que les sous-groupes $t_{\mathbb{Z}_{p}} M_{\Gamma} / p$ et $t_{\mathbb{Z}_{p}} M(1)_{\Gamma} / p$ de $M_{\Gamma} / p$ sont égaux, bien que l'inégalité $h^{\prime}+k \leq n+v_{p}(e)$ ne soit pas satisfaite. En fait $Z=\mathbb{Z}_{p}[[T]] /\left(T^{2}, p T, p^{2}\right)$ et $Z^{\prime}=Z^{\Gamma}=p Z+T Z=\mathbb{Z} / p \oplus \mathbb{Z} / p$, le raisonnement de la preuve ci-dessus donne donc seulement $n+v_{p}(e) \geq v_{p}\left(\exp \left(Z^{\prime}\right)\right)=1$.

Remarque 2.8. - Le théorème 2.2 est de nature asymptotique. Cela tient au choix, suivant [G], de l'objet $X^{\prime}\left(F_{\infty}\right)^{0}$ pour mesurer la différence entre les noyaux de Tate. Dans [V3], nous remplacerons $X^{\prime}\left(F_{\infty}\right)^{0}$ par un objet au niveau fini, ce qui donnera lieu à des résultats au niveau fini.

\section{Comparaison $F$-rationnelle}

Soit $E / F$ une extension galoisienne, et $i$ et $j$ deux entiers $E$-bons, comparables. Si le noyau de capitulation est trivial, alors d'après le théorème 2.2 , on a $k(E, i, j)=m(E)+v_{p}(i-j)$, en d'autres termes, les $i^{\text {ème }}$ et $j$ ème noyaux de Tate sont égaux avec la meilleure précision que l'on puisse espérer. Dans la pratique, il arrive souvent qu'on ait à faire varier le corps $E$, si bien que dans l'état actuel de nos connaissances, l'hypothèse selon laquelle $i$ et $j$ sont $E$-bons n'est raisonnable que si $i$ et $j$ sont tous deux $\geq 2$ (thm. de Soulé). L'objet de ce paragraphe est de montrer que

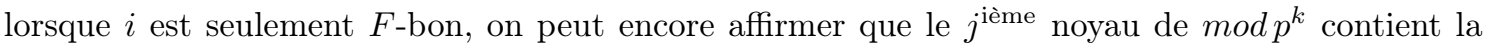
partie $F$-rationnelle (voir ci-dessous) du $i^{\text {ème }}$ noyau de Tate $\bmod p^{k}$.

Définition 3.1. — Si E/F est une extension galoisienne finie, on définit le sous-groupe Frationnel $V_{i}\left(E / F, p^{k}\right)$ de $V_{i}\left(E, p^{k}\right)$ par

$$
V_{i}\left(E / F, p^{k}\right)=\operatorname{Im}\left(H_{S}^{1}\left(E, \mathbb{Z}_{p}(i)\right)^{G(E / F)} \rightarrow H_{S}^{1}\left(E, \mathbb{Z} / p^{k}(i)\right)\right)
$$

Notons que si $i \neq 0, V_{i}\left(E / F, p^{k}\right)$ est simplement $\operatorname{res}_{F}^{E} V_{i}\left(F, p^{k}\right)$, mais si $i=0$, ce n'est plus nécessairement le cas. En fait $V_{0}\left(E / F, p^{k}\right)$ décrit les premiers étages des $\mathbb{Z}_{p}$-extensions de $E$ qui proviennent de $F$ par composition avec $E$.

Théorème 3.2. - Soit $E / F$ une sous-extension finie de $\tilde{F} / F$. On suppose $i F$-bon, et $j \equiv$ $i \bmod \left[F\left(\mu_{p}\right): F\right]$. Si $X^{(i)}\left(E_{\infty}\right)^{0}=0$, alors $V_{i}\left(E / F, p^{k}\right) \subset V_{j}\left(E, p^{k}\right)$ pour tout $k \leq m(F)+v_{p}(i-j)$.

Preuve : Comme $j \equiv i \bmod \left[F\left(\mu_{p}\right): F\right]$, on peut identifier les groupes $H_{S}^{1}\left(E_{\infty}, \mathbb{Q}_{p} / \mathbb{Z}_{p}(i)\right)$ et $H_{S}^{1}\left(E_{\infty}, \mathbb{Q}_{p} / \mathbb{Z}_{p}(j)\right)$. Alors, d'après le lemme 2.3 , on a

$$
V_{i}\left(E / F, p^{k}\right) \subset V_{j}\left(E, p^{k}\right) \Leftrightarrow \operatorname{res}_{F}^{E_{\infty}} V_{i}\left(E / F, p^{k}\right) \subset\left(H_{S}^{1}\left(E_{\infty}, \mathbb{Q}_{p} / \mathbb{Z}_{p}(j)\right)^{\Gamma}\right)^{d i v}
$$

Considérons l'accouplement suivant :

$$
H_{S}^{1}\left(E_{\infty}, \mathbb{Q}_{p} / \mathbb{Z}_{p}(j)\right)^{\Gamma} \otimes \mathcal{X}^{(-j)}\left(E_{\infty}\right)_{\Gamma} \longrightarrow \mathbb{Q}_{p} / \mathbb{Z}_{p}
$$

Comme il s'agit d'un accouplement parfait, on montre que l'orthogonal de $\operatorname{res}_{F}^{E_{\infty}} V_{i}\left(E / F, p^{k}\right)$ contient celui de $\left(H_{S}^{1}\left(E_{\infty}, \mathbb{Q}_{p} / \mathbb{Z}_{p}(j)\right)^{\Gamma}\right)^{d i v}$. Comme $H^{1}\left(\Gamma, f_{\Lambda} \mathcal{X}^{(-j)}\left(E_{\infty}\right)\right)=\left(f_{\Lambda} \mathcal{X}^{(-j)}\left(E_{\infty}\right)\right)^{\Gamma}=0$, on peut considérer $\left(t_{\Lambda} \mathcal{X}^{(-j)}\left(E_{\infty}\right)\right)_{\Gamma}$ comme un sous-espace de $\mathcal{X}^{(-j)}\left(E_{\infty}\right)_{\Gamma}$. Nous montrerons les deux faits suivants :

Fait 1 : L'orthogonal de $\operatorname{res}_{F}^{E_{\infty}} V_{i}\left(E / F, p^{k}\right)$ contient $\left(t_{\Lambda} \mathcal{X}^{(-j)}\left(E_{\infty}\right)\right)_{\Gamma}$.

Fait 2 : L'orthogonal de $\left(H_{S}^{1}\left(E_{\infty}, \mathbb{Q}_{p} / \mathbb{Z}_{p}(j)\right)^{\Gamma}\right)^{\text {div }}$ est contenu dans $\left(t_{\Lambda} \mathcal{X}^{(-j)}\left(E_{\infty}\right)\right)_{\Gamma}$.

Commençons par le fait 1 . Il s'agit de montrer que $\operatorname{res}_{F}^{E_{\infty}} V_{i}\left(E / F, p^{k}\right)$ et $t_{\Lambda} \mathcal{X}^{(-j)}\left(E_{\infty}\right)$ sont orthogonaux pour l'accouplement

$$
H_{S}^{1}\left(E_{\infty}, \mathbb{Q}_{p} / \mathbb{Z}_{p}(j)\right) \otimes \mathcal{X}^{(-j)}\left(E_{\infty}\right) \longrightarrow \mathbb{Q}_{p} / \mathbb{Z}_{p}
$$


Notons d'abord l'inclusion suivante

$$
\operatorname{res}_{E}^{E_{\infty}} V_{i}\left(E / F, p^{k}\right) \subset \operatorname{res}_{F}^{E_{\infty}}\left(H_{S}^{1}\left(F, \mathbb{Q}_{p} / \mathbb{Z}_{p}(i)\right)^{d i v}\right)
$$

(l'inclusion a lieu dans $\left.H_{S}^{1}\left(E_{\infty}, \mathbb{Q}_{p} / \mathbb{Z}_{p}(i)\right)\right)$. En effet, $\operatorname{res}_{E}^{E_{\infty}} V_{i}\left(E / F, p^{k}\right)$ est l'image de l'application composée

$$
H_{S}^{1}\left(E, \mathbb{Z}_{p}(i)\right)^{G(E / F)} \rightarrow H_{S}^{1}\left(E, \mathbb{Z} / p^{k}(i)\right) \stackrel{r e s_{F}^{E}}{\rightarrow} H_{S}^{1}\left(E_{\infty}, \mathbb{Z} / p^{k}(i)\right) \stackrel{\frac{1}{p^{k}}}{\rightarrow} H_{S}^{1}\left(E_{\infty}, \mathbb{Q}_{p} / \mathbb{Z}_{p}(i)\right)
$$

que l'on peut ré-écrire

$$
H_{S}^{1}\left(E, \mathbb{Z}_{p}(i)\right)^{G(E / F)} \rightarrow H_{S}^{1}\left(E, \mathbb{Z} / p^{k}(i)\right) \stackrel{\frac{1}{p^{k}}}{\rightarrow} H_{S}^{1}\left(E, \mathbb{Q}_{p} / \mathbb{Z}_{p}(i)\right) \stackrel{r e s_{E}^{E}}{\rightarrow} H_{S}^{1}\left(E_{\infty}, \mathbb{Q}_{p} / \mathbb{Z}_{p}(i)\right)
$$

ou encore

$$
H_{S}^{1}\left(E, \mathbb{Z}_{p}(i)\right)^{G(E / F) \stackrel{\frac{1}{p^{k}}}{\rightarrow}} H_{S}^{1}\left(E, \mathbb{Q}_{p}(i)\right)^{G(E / F)} \rightarrow H_{S}^{1}\left(E, \mathbb{Q}_{p} / \mathbb{Z}_{p}(i)\right) \stackrel{r e s_{E}^{E}}{\rightarrow} H_{S}^{1}\left(E_{\infty}, \mathbb{Q}_{p} / \mathbb{Z}_{p}(i)\right) .
$$

Avec l'égalité $H_{S}^{1}\left(E, \mathbb{Q}_{p}(i)\right)^{G(E / F)}=\operatorname{res}_{F}^{E} H_{S}^{1}\left(F, \mathbb{Q}_{p}(i)\right)$, on obtient l'inclusion du sous-groupe $\operatorname{res}_{E}^{E_{\infty}} V_{i}\left(E / F, p^{k}\right)$ de $H_{S}^{1}\left(E, \mathbb{Q}_{p} / \mathbb{Z}_{p}(i)\right)$ dans l'image de l'application composée suivante

$$
H_{S}^{1}\left(F, \mathbb{Q}_{p}(i)\right) \stackrel{r e s_{F}^{E}}{\rightarrow} H_{S}^{1}\left(E, \mathbb{Q}_{p}(i)\right) \rightarrow H_{S}^{1}\left(E, \mathbb{Q}_{p} / \mathbb{Z}_{p}(i)\right) \stackrel{r e s_{E}^{E}}{\rightarrow} H_{S}^{1}\left(E, \mathbb{Q}_{p} / \mathbb{Z}_{p}(i)\right)
$$

que nous ré-écrivons

$$
H_{S}^{1}\left(F, \mathbb{Q}_{p}(i)\right) \rightarrow H_{S}^{1}\left(F, \mathbb{Q}_{p} / \mathbb{Z}_{p}(i)\right) \stackrel{r e s_{F}^{E}}{\rightarrow} H_{S}^{1}\left(E, \mathbb{Q}_{p} / \mathbb{Z}_{p}(i)\right)
$$

D'où l'inclusion (4), puisque $H_{S}^{1}\left(F, \mathbb{Q}_{p}(i)\right)$ est divisible.

Maintenant, l'accouplement (3) est compatible avec le suivant :

$$
H_{S}^{1}\left(F, \mathbb{Q}_{p} / \mathbb{Z}_{p}(i)\right) \otimes \mathcal{X}^{(-i)}(F) \longrightarrow \mathbb{Q}_{p} / \mathbb{Z}_{p}
$$

(noter que l'on peut remplacer $j$ par $i$ dans (3)) Ainsi pour montrer que $r e s_{F}^{E_{\infty}} V_{i}\left(E / F, p^{k}\right)$ et $t_{\Lambda} \mathcal{X}^{(-j)}\left(E_{\infty}\right)$ (comme groupe c'est $t_{\Lambda} \mathcal{X}^{(-i)}\left(E_{\infty}\right)$ ) sont orthogonaux, il suffit de montrer que l'image de $t_{\Lambda} \mathcal{X}^{(-i)}\left(E_{\infty}\right) \rightarrow \mathcal{X}^{(-i)}(F)$ est orthogonale à $H_{S}^{1}\left(F, \mathbb{Q}_{p} / \mathbb{Z}_{p}(i)\right)^{\text {div }}$ pour l'accouplement (5). Remarquons pour cela que l'application $t_{\Lambda} \mathcal{X}^{(-i)}\left(E_{\infty}\right) \rightarrow \mathcal{X}^{(-i)}(F)$ se factorise en

$$
t_{\Lambda} \mathcal{X}^{(-i)}\left(E_{\infty}\right) \rightarrow t_{\Lambda} \mathcal{X}^{(-i)}\left(F_{\infty}\right) \rightarrow\left(t_{\Lambda} \mathcal{X}^{(-i)}\left(F_{\infty}\right)\right)_{\Gamma} \rightarrow \mathcal{X}^{(-i)}(F)
$$

Comme $i$ est $F$-bon, $\left(t_{\Lambda} \mathcal{X}^{(-i)}\left(F_{\infty}\right)\right)_{\Gamma}$ est fini (cf [V3], Cor. 1.2.41), l'image l'est donc aussi de $t_{\Lambda} \mathcal{X}^{(-i)}\left(E_{\infty}\right)$ dans $\mathcal{X}^{(-i)}(F)$ l'est donc aussi, et cela entraîne l'orthogonalité souhaitée.

Passons au fait 2. L'orthogonal de $\left(H_{S}^{1}\left(E_{\infty}, \mathbb{Q}_{p} / \mathbb{Z}_{p}(j)\right)^{\Gamma}\right)^{\text {div }}$ est exactement $t_{\mathbb{Z}_{p}}\left(\mathcal{X}^{(-j)}\left(E_{\infty}\right)_{\Gamma}\right)$. Comme $X^{(j)}\left(E_{\infty}\right)^{0}=0$, c'est que $f_{\Lambda} \mathcal{X}^{(-j)}\left(E_{\infty}\right)$ est libre (cf [V3], rem. 3.2.27), et donc que $t_{\mathbb{Z}_{p}}\left(\mathcal{X}^{(-j)}\left(E_{\infty}\right)_{\Gamma}\right) \subset\left(t_{\Lambda} \mathcal{X}^{(-j)}\left(E_{\infty}\right)\right)_{\Gamma}$

\section{Références}

[AM] J. Assim, A. Movahhedi, Bounds for Étale Capitulation Kernels, K-Theory, 33 (2004), 199-213.

[H] K. Hutchinson, Tate kernels, étale K-theory and the Gross Kernel, Preprint (2005).

[KM] M. Kolster, A. Movahhedi, Galois co-descent for étale wild kernels and capitulation, Ann. Inst. Fourier, Grenoble 50 (2000), 35-65.

[G] R. Greenberg, A note on $K_{2}$ and the theory of $\mathbb{Z}_{p}$-extensions, Amer. J. of Math. 100, No.6. (1978), 1235-1245.

[J] U. Jannsen, Iwasawa Modules up to Isomorphism, Advanced Studies in Pure Mathematics 17 (1989), 171-207.

[S] C. Soulé, K-théorie des anneaux d'entiers de corps de nombres et cohomologie étale, Invent. Math. 55 (1979), 251-295.

[KC] K. Kramer et A. Candiotti, On $K_{2}$ and $\mathbb{Z}_{l}$-extensions of number fields, Amer. Journal of Math. 100 (1978), 177-196).

[T] J. Tate, Relations between $K_{2}$ and Galois cohomology, Invent. Math. 36 (1976), 257-274. 
[V1] D. Vauclair, Cup produit, noyaux de capitulations étales et conjecture de Greenberg généralisée, Ktheory, 36 (2005), 223-244.

[V2] D. Vauclair, Noyaux de Tate et capitulation, en préparation (2006).

[V3] D. Vauclair, Conjecture de Greenberg généralisée et capitulation dans les $\mathbb{Z}_{p}$-extensions d'un corps de nombres, thèse (2005).

8 novembre 2006

David Vauclair, Université de Franche-Comté, Laboratoire de Mathématiques, CNRS UMR 6623, 16, route de Gray, 25030 BESANCON CEDEX, France $\bullet$ E-mail : vauclair@math.univ-fcomte.fr 